EPJ Web of Conferences 81, 04004 (2014)

DOI: 10.1051/epjconf/20148104004

(C) Owned by the authors, published by EDP Sciences, 2014

\title{
Hadronic resonance production measured with the ALICE detector at the LHC
}

\author{
Enrico Fragiacomo ${ }^{1, a}$ \\ for the ALICE Collaboration \\ ${ }^{1}$ Istituto Nazionale di Fisica Nucleare
}

\begin{abstract}
Hadronic resonance production has been studied with the ALICE detector in different collision systems at LHC energies. The aim is to disentangle initial-state effects from genuine medium-induced effects, which may occur in the hot and dense hadronic matter after the chemical freeze-out in $\mathrm{Pb}-\mathrm{Pb}$ collisions. In these proceedings, transversemomentum spectra of $\phi(1020)$ and $\mathrm{K}^{*}(892)^{0}$ and the ratio of their yield to long-lived particles are discussed in this light.
\end{abstract}

\section{Introduction}

In ultra-relativistic heavy-ion collisions, a hot and dense state of matter, the quark-gluon plasma, is produced [1], which is expected to undergo a transition from partonic to hadronic phase (the so-called chemical freeze-out) at a predicted temperature of $T_{c} \simeq 156 \mathrm{MeV}$ [2]. Particle yields at chemical freeze-out have been found to be successfully predicted by statistical models, but the measured resonance yields may deviate from the predictions because hadronic processes such as regeneration and re-scattering, which affect the short-lived resonances, occur in the hadronic phase before kinetic freeze-out [3]. Therefore, reconstructing resonances in their hadronic decay channel can provide some insight into the dynamical evolution of the fireball between chemical and kinetic freeze-out.

Both meson and baryon resonances have been measured by the ALICE experiment [4] in different collision systems (pp, p- $\mathrm{Pb}, \mathrm{Pb}-\mathrm{Pb}$ ) at LHC energies [5-8]. The study of resonance production in $\mathrm{pp}$ and $\mathrm{p}-\mathrm{Pb}$ collisions provides a necessary baseline to disentangle initial-state effects from genuine medium-induced effects, which may occur in $\mathrm{Pb}-\mathrm{Pb}$ collisions. In these proceedings, focus is given to the meson resonances $\phi(1020)$ and $\mathrm{K}^{*}(892)^{0}$, reconstructed at mid-rapidity in $\mathrm{Pb}-\mathrm{Pb}$ collisions at $\sqrt{s_{N N}}=2.76 \mathrm{TeV}[5]$ and in $\mathrm{p}-\mathrm{Pb}$ collisions at $\sqrt{s_{N N}}=5.02 \mathrm{TeV}$. Transverse-momentum distributions and particle ratios to stable particles as function of the multiplicity are discussed in Section 2 and 3, respectively, suggesting that in central $\mathrm{Pb}-\mathrm{Pb}$ collisions $\mathrm{K}^{* 0}$ suffers from re-scattering due to its short lifetime $(\tau \simeq 4 \mathrm{fm} / c)$, while compared to it, $\phi(\tau \simeq 45 \mathrm{fm} / c)$ behaves as a long lived particle.

ae-mail: enrico.fragiacomo@ts.infn.it 


\section{Transverse momentum spectra}

The transverse momentum distributions of the $\phi$ and $\mathrm{K}^{* 0}$ resonances in central (0-20\%) and peripheral $(60-80 \%) \mathrm{Pb}-\mathrm{Pb}$ collisions at $\sqrt{s_{N N}}=2.76 \mathrm{TeV}$ [5] are compared in Figure 1 to the blast-wave [9] prediction for the spectral shapes. The parameters of the blast-wave curves are obtained from a simultaneous fit to the transverse-momentum distributions of charged particles (pions, kaons and protons) in $\mathrm{Pb}-\mathrm{Pb}$ collisions [10]. The curves are normalized to the measured yields of the charged kaons in $\mathrm{Pb}-\mathrm{Pb}$ collisions [10], multiplied by the $\phi / \mathrm{K}$ and $\mathrm{K}^{* 0} / \mathrm{K}$ ratios given by a thermal-model fit to ALICE data [2]. This provides the expected yields if no re-scattering effects occur.

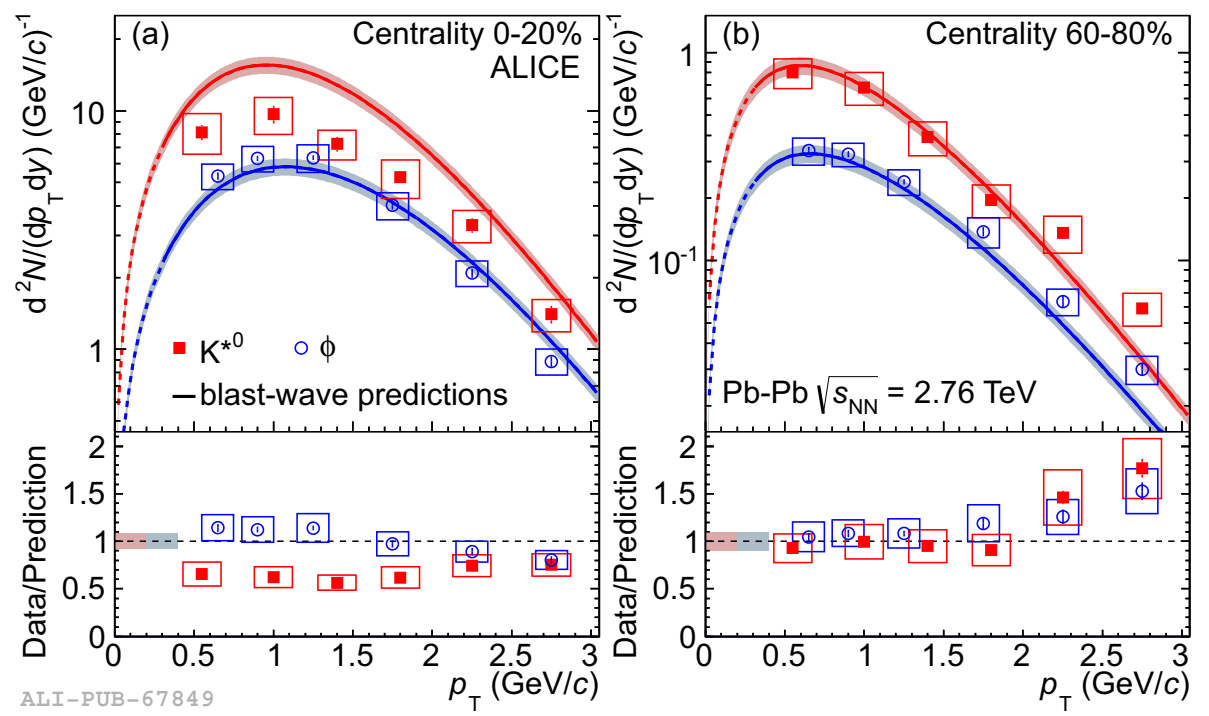

Figure 1. Transverse-momentum distributions of $\phi$ and $\mathrm{K}^{* 0}$ resonances in central (a) and peripheral (b) $\mathrm{Pb}-\mathrm{Pb}$ collisions at $\sqrt{s_{N N}}=2.76 \mathrm{TeV}$ [5], compared to blast-wave predictions of the shapes, without re-scattering effects. Statistical and systematic uncertainties are shown as bars and boxes, respectively. The shaded bands indicate the uncertainties in the normalization of the predicted distributions. The lower panels show the ratios of the measured distributions to the predictions.

For the $\phi$ meson the predictions are satisfactorily describing the data over all the measured $p_{\mathrm{T}}$ range, in both central and peripheral collisions. The data/theory ratio is around unity up to $p_{\mathrm{T}} \simeq 2 \mathrm{GeV} / c$. The same conclusions hold for the $\mathrm{K}^{* 0}$ in peripheral collisions, where the data/theory ratio does not appear to deviate significantly from unity for $p_{\mathrm{T}}<2 \mathrm{GeV} / c$. On the contrary, in central collisions the $\mathrm{K}^{* 0}$ appears suppressed by a factor $\gtrsim 1 / 3$. The deviation is about three times larger than the uncertainties, giving some hints that $\mathrm{K}^{* 0}$ has undergone non-negligible re-scattering effects.

\section{Particle ratios}

Figure 2 shows the ratios of particle yields, $\mathrm{K}^{* 0} / \mathrm{K}^{-}$and $\phi / \mathrm{K}^{-}$for $\mathrm{Pb}-\mathrm{Pb}$ collisions at $\sqrt{s_{N N}}=2.76 \mathrm{TeV}$ [5], p-Pb collisions at $\sqrt{s_{N N}}=5.02 \mathrm{TeV}$ and pp collisions at $\sqrt{s}=7 \mathrm{TeV}$ [7]. These ratios are presented as function of $\left(\mathrm{d} N_{c h} / \mathrm{d} \eta\right)^{1 / 3}$, where $\mathrm{d} N_{c h} / \mathrm{d} \eta$ is the charged-particle multiplicity density. The cube root relates $\mathrm{d} N_{c h} / \mathrm{d} \eta$ to the system radius [11]. 


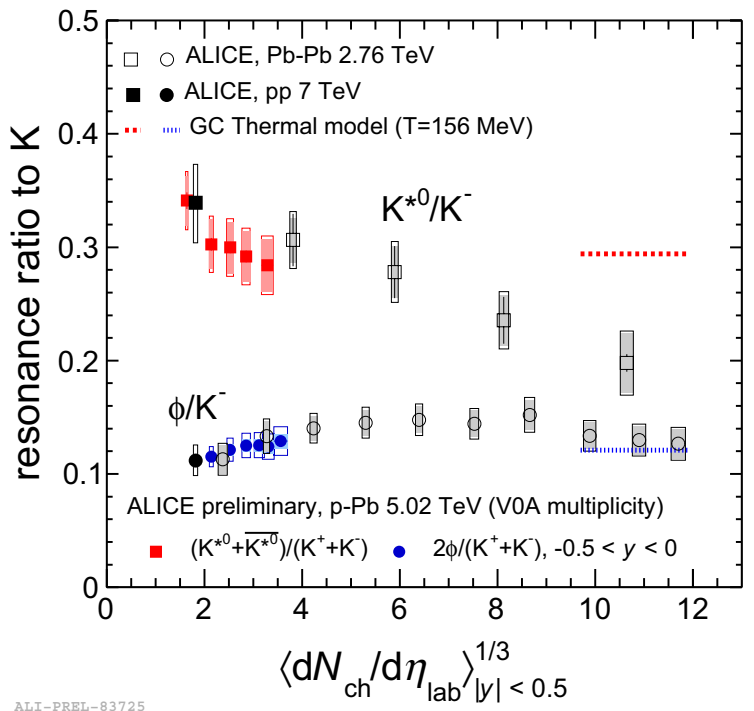

Figure 2. Ratios of particle yields, $\mathrm{K}^{* 0} / \mathrm{K}^{-}$and $\phi / \mathrm{K}^{-}$, as function of the cube root of the charged-particle multiplicity density, $\mathrm{d} N_{c h} / \mathrm{d} \eta[5,7]$. Thermal model predictions [2] are given for central $\mathrm{Pb}-\mathrm{Pb}$ collisions.

The $\phi / \mathrm{K}^{-}$ratio in central $\mathrm{Pb}-\mathrm{Pb}$ collisions is consistent with the values measured in $\mathrm{pp}$ and $\mathrm{p}-\mathrm{Pb}$ collisions, as well as with the prediction of a thermal model [2], which has a chemical freeze-out temperature of $156 \mathrm{MeV}$ and a baryochemical potential of $0 \mathrm{MeV}$ and does not include re-scattering effects. The parameters of the model are obtained by fitting particle yields measured in central $\mathrm{Pb}-\mathrm{Pb}$ collisions, including the $\phi$ yield and excluding the $\mathrm{K}^{* 0}$ yield. The $\mathrm{K}^{* 0} / \mathrm{K}^{-}$ratio in central $\mathrm{Pb}-\mathrm{Pb}$ collisions appears significantly lower than in peripheral collisions, as well as in $\mathrm{pp}$ and $\mathrm{p}-\mathrm{Pb}$ collisions. This suppression of the $\mathrm{K}^{* 0} / \mathrm{K}^{-}$ratio in more central $\mathrm{Pb}-\mathrm{Pb}$ collisions may be related to re-scattering effects, which affect only the $\mathrm{K}^{* 0}$ meson due to its shorter lifetime compared to the lifetime of the fireball.

\section{References}

[1] U. W. Heinz and M. Jacob, nucl-th/0002042.

[2] J. Stachel et al., J. Phys.: Conf. Ser. 509, 012019 (2014).

[3] B. Abelev et al. (STAR Collaboration), Phys. Rev. Lett. 97, 132301 (2006).

[4] K. Aamodt et al. (ALICE Collaboration), J. Instrum. 3, S08002 (2008).

[5] B. Abelev et al. (ALICE Collaboration), submitted to Phys. Rev. C, arXiv:1404.0495.

[6] B. Abelev et al. (ALICE Collaboration), Eur. Phys. J. C 71, 1594 (2011).

[7] B. Abelev et al. (ALICE Collaboration), Eur. Phys. J. C 72, 2183 (2012).

[8] B. Abelev et al. (ALICE Collaboration), submitted to Eur. Phys. J. C, arXiv:1406.3206.

[9] E. Schnedermann, J. Sollfrank and U. Heinz, Phys. Rev. C 48, 2462 (1993).

[10] B. Abelev et al. (ALICE Collaboration), Phys. Lett. B 728, 2538 (2014).

[11] K. Aamodt et al. (ALICE Collaboration), Phys. Lett. B 696, 328 (2011).

[12] B. Abelev et al. (ALICE Collaboration), Phys. Rev. C 88, 044910 (2013). 